\title{
Tensile mechanical behavior and fracture toughness of MWCNT and DWCNT modified vinyl-ester/polyester hybrid nanocomposites produced by 3-roll milling
}

\author{
A. Tuğrul Seyhan a , Metin Tanoğlu ${ }^{\mathrm{b}, *}$, Karl Schulte $^{\mathrm{c}}$ \\ a Department of Materials Science and Engineering, Anadolu University (AU), Iki Eylul Campus, 26550 Eskisehir, Turkey \\ ${ }^{\mathrm{b}}$ Izmir Institute of Technology (IZTECH), Mechanical Engineering Department, Gulbahce Campus 35437, Izmir, Turkey \\ ${ }^{\text {c } T e c h n i c s h e ~ U n i v e r s i t a t ~ H a m b u r g-H a r b u r g ~(T U H H), ~ P o l y m e r ~ C o m p o s i t e s ~ S e c t i o n, ~ D e n i c k e s t r a s s e ~ 15, ~ D-21073 ~ H a m b u r g, ~ G e r m a n y ~}$
}

\section{A R T I C L E I N F O}

\section{Article history:}

Received 18 March 2009

Received in revised form 17 May 2009

Accepted 18 May 2009

\section{Keywords:}

Carbon nanotubes (CNTs)

Thermosetting resins

3-Roll milling

Tensile behavior

Fracture toughness

Transmission Electron Microscopy (TEM)

\begin{abstract}
A B S T R A C T
This study aims to investigate the tensile mechanical behavior and fracture toughness of vinylester/polyester hybrid nanocomposites containing various types of nanofillers, including multi- and double-walled carbon nanotubes with and without amine functional groups (MWCNTs, DWCNTs, MWCNT-NH $\mathrm{N}_{2}$ and DWCNT-NH$\left.{ }_{2}\right)$. To prepare the resin suspensions, very low contents $(0.05,0.1$ and $0.3 \mathrm{wt} . \%$ ) of carbon nanotubes (CNTs) were dispersed within a specially synthesized styrene-free polyester resin, conducting 3-roll milling technique. The collected resin stuff was subsequently blended with vinyl-ester via mechanical stirring to achieve final suspensions prior to polymerization. Nanocomposites containing MWCNTs and MWCNT- $\mathrm{NH}_{2}$ were found to exhibit higher tensile strength and modulus as well as larger fracture toughness and fracture energy compared to neat hybrid polymer. However, incorporation of similar contents of DWCNTs and DWCNT- $\mathrm{NH}_{2}$ into the hybrid resin did not reflect the same improvement in the corresponding mechanical properties. Furthermore, experimentally measured elastic moduli of the nanocomposites containing DWCNTs, DWCNT- $\mathrm{NH}_{2}$, MWCNTs and MWCNT-NH $\mathrm{N}_{2}$ were fitted to Halphin-Tsai model. Regardless of amine functional groups or content of carbon nanotubes, MWCNT modified nanocomposites exhibited better agreement between the predicted and the measured elastic moduli values compared to nanocomposites with DWCNTs. Furthermore, Transmission Electron Microscopy (TEM) and Scanning Electron Microscopy (SEM) were used to reveal dispersion state of the carbon nanotubes within the hybrid polymer and to examine the CNT induced failure modes that occurred under mechanical loading, respectively. Based on the experimental findings obtained, it was emphasized that the types of CNTs and presence of amine functional groups on the surface of CNTs affects substantially the chemical interactions at the interface, thus tuning the ultimate mechanical performance of the resulting nanocomposites.
\end{abstract}

(C) 2009 Elsevier B.V. All rights reserved.

\section{Introduction}

Nano-particles have recently gained great interest in science and industry due to their highly considerable promises in enabling the future nano-structured materials with novel properties [1,6]. In this respect, carbon nanotubes (CNTs) with their huge aspect ratio in combination with high strength and stiffness have become potential reinforcing constituents to get common engineering polymers into multi-functional composites with superior properties such as conductive polymers with improved mechanical performance [1,5]. Despite many reported studies in the literature, the achievement of the desired improvement via CNTs in final properties of their polymer based composites has not been successfully realized so far [2,7].

\footnotetext{
* Corresponding author. Fax: +90 2327507890.

E-mail address: metintanoglu@iyte.edu.tr (M. Tanoğlu).
}

This compromise arises primarily from the weak interfacial bonding of CNTs with the surrounding polymer matrices because of their very inert surfaces as well as their non-homogeneous dispersion within polymers due to their huge surface area [5,8]. To promote the compatibility at the interface between CNTs and the surrounding polymer matrix, some chemical functional groups are applied over the surfaces of CNTs [4,8]. Kim et al. [7] found that addition of untreated, acid and $\mathrm{NH}_{2}$ treated multi-walled carbon nanotubes (MWCNTs) into epoxy resin resulted in 61, 69 and 73\% increase, respectively, in tensile strength of the resulting nanocomposites as compared to neat epoxy resin, without significantly reducing the elastic modulus of the nanocomposites. Yaping et al. [8] investigated the effect of MWCNT- $\mathrm{NH}_{2}$ on the mechanical properties of the epoxy resin. They stated that flexural bending stress and modulus of the nanocomposites were significantly improved as compared to those of neat epoxy resin. Moreover, in the same study, impact strength of the corresponding nanocomposites was measured to 
be two times higher than that of neat epoxy resin. Grujicic et al. [9] studied the atomic level mechanical properties of three-walled carbon nanotubes ( 3 WCNTs) reinforced vinyl-ester resin with epoxy group, using molecular mechanics to interpret the effect of covalent functionalization. They revealed that covalent functionalization has a profound effect on the nanocomposites as the load is applied in a direction orthogonal to nanotube axis. Homogeneous dispersion of CNTs within polymers is another crucial issue to observe the desired final properties in the resultant nanocomposites [5,8]. Strong Van der Waals forces and interactions between relatively small diameter nanotubes lead to aggregates of nanotube ropes to exist $[1,9]$. Therefore, length of the tubes, their degree of entanglement and volume fraction as well as the viscosity of the surrounding matrix resin are critical to dispersion state of CNTs within the matrix resin. A number of different techniques including high speed mechanical stirring and sonication are commonly used for proper dispersion of CNTs within polymers $[3,5-9,10,13,16]$. Of all, sonication has been the most widely utilized technique for $\mathrm{CNT} /$ thermosetting resin systems such as epoxy and vinyl-ester. In this technique, a pulsed ultrasound with certain amplitude is intended for uniform dispersion of CNTs by dividing their agglomerates into individuals or smaller ones. However, the magnitude of vibration energy applied is limited to distance from the sonicator tip. Therefore, it is not scalable from laboratory to industrial manufacturing settings and not cost effective any more. Gojny et al. [2,4] alternatively utilized the 3-roll milling to accomplish relatively good dispersion of nanotubes with and without $\mathrm{NH}_{2}$ functional group within epoxy resins. They revealed based upon TEM investigations that double-walled carbon nanotubes (DWCNTs) have moderately fine dispersion in epoxy resins via 3-roll milling. In the same study, they also showed that blending of very low content ( $0.1 \mathrm{wt}$.\%) of DWCNTs with epoxy resin via 3-roll milling improved the mechanical properties, such as elastic modulus and fracture toughness of the polymers. Thostenson and Chou [16] investigated recently the influence of processing parameters on the final dispersion state of CNTs during 3-roll milling. In this manner, they produced epoxy/CNT suspensions at various gap settings ranging from subsequently 5 to $50 \mu \mathrm{m}$ through 3-roll milling by means of Teflon guides which help keep the blend centered on the rollers. They observed that nanocomposites processed at gap settings of 5 and $10 \mu \mathrm{m}$ have relatively high fracture toughness values.

In the present study, 3-roll milling was used to prepare vinylester-polyester hybrid resin based nanocomposites containing various contents of MWCNT, MWCNT-NH 2 , DWCNT and DWCNT$\mathrm{NH}_{2}$. To produce the nanocomposites, the CNTs were first dispersed within specially synthesized styrene-free polyester resin followed by blending the collected resin stuff with vinyl-ester resin via mechanical stirring. The dispersion state of the CNTs within the resulting nanocomposites was revealed via Transmission Electron Microscopy (TEM) studies. The influence of CNTs upon the tensile mechanical response and fracture toughness of the nanocomposites were comprehensively evaluated in association with the fracture modes obtained via SEM examination.

\section{Experimental}

\subsection{Materials}

Thin MWCNTs and DWCNTs with and without $\mathrm{NH}_{2}$ functional groups were purchased from Nanocyl (Namur Belgium) and utilized as reinforcing constituents for the corresponding hybrid resin. Table 1 gives the physical properties of the corresponding CNTs used in this study. Styrene-free polyester resin POLIYA 420 and vinylester-epoxy resin (bisphenol A epoxy based) POLIPOL 701 with $35 \mathrm{wt}$.\% of styrene were provided from POLIYA Polyester, Turkey.
Table 1

Physical property of carbon nanotubes.

\begin{tabular}{|c|c|c|c|c|}
\hline Nanotube types & $P_{C N T}$ & $L$ & $d_{\text {in }}$ & $d_{\text {out }}$ \\
\hline DWCNT & $0.98 \mathrm{~g} / \mathrm{cm}^{3}$ & $10 \mu \mathrm{m}$ & $2.1 \mathrm{~nm}$ & $2.8 \mathrm{~nm}$ \\
\hline DWCNT-NH ${ }_{2}$ & $0.98 \mathrm{~g} / \mathrm{cm}^{3}$ & $5 \mu \mathrm{m}$ & $2.1 \mathrm{~nm}$ & $2.8 \mathrm{~nm}$ \\
\hline MWCNT & $2.09 \mathrm{~g} / \mathrm{cm}^{3}$ & $50 \mu \mathrm{m}$ & $4 \mathrm{~nm}$ & $15 \mathrm{~nm}$ \\
\hline MWCNT-NH & $2.09 \mathrm{~g} / \mathrm{cm}^{3}$ & $10 \mu \mathrm{m}$ & $4 \mathrm{~nm}$ & $15 \mathrm{~nm}$ \\
\hline
\end{tabular}

Styrene emission agent BKY 740, purchased from Alton Chemie, Germany, was utilized to prevent evaporation of styrene during polymerization reaction. To polymerize the resin suspensions, cobalt naphtanate (CoNAP) and methyl ethyl kethone peroxide (MEKP) were then used as accelerator and initiator, respectively.

\subsection{Principles of 3-roll milling}

3-Roll milling is highly capable of dispersing CNTs homogeneously within thermosetting polymers without leading to a rupture and damage of CNTs that reduce their aspect ratio, unlike other solvent based techniques or treatments do [2,4]. Fig. 1 shows the photo of resin suspension on the rolls at $30 \mathrm{~s}$ after it was fed. 3-Roll milling differs principally from other types of mills in that it applies almost pure shear rather than compressive load. The first and third rolls labeled in Fig. 1 are usually called feed and apron rolls, respectively. They rotate in the same direction, while the center roll (2) rotates in the opposite direction. In order to create high shear rates, angular velocity of the center roll must be higher than that of feed roll $(w 2>w 1)[2,14]$. As the resin suspension is fed into the narrow gap between feed and center rolls, the liquid stuff flows down covering the adjacent rolls through its surface tension under intensive shear forces. At the end of each subsequent intended dwell time, the processed resin suspension is collected by using a scraper blade in contact with the apron roll.

\subsection{Preparation of nanocomposites}

Various amounts of MWCNTs, MWCNT- $\mathrm{NH}_{2}$ DWCNTs, DWCNT$\mathrm{NH}_{2}(0.05,0.1$ and $0.3 \mathrm{wt} . \%)$ were first mixed manually with styrene-free polyester resin prior to 3-roll milling processing for $10 \mathrm{~min}$. A 3-roll mill (Exakt $120 \mathrm{~S}$ Exakt $\mathrm{Gmbh}$ ) with alumina ceramic rolls was used to disperse the CNTs within styrene-free

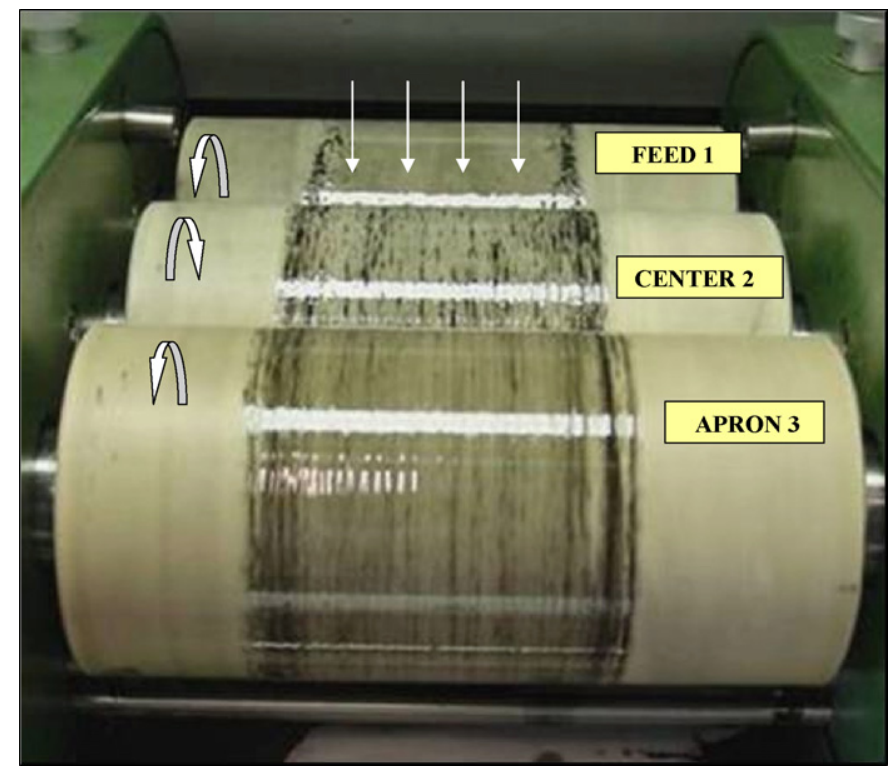

Fig. 1. Nanotube hybrid resin suspension on the rolls at time $t=30 \mathrm{~s}$ after it was fed 

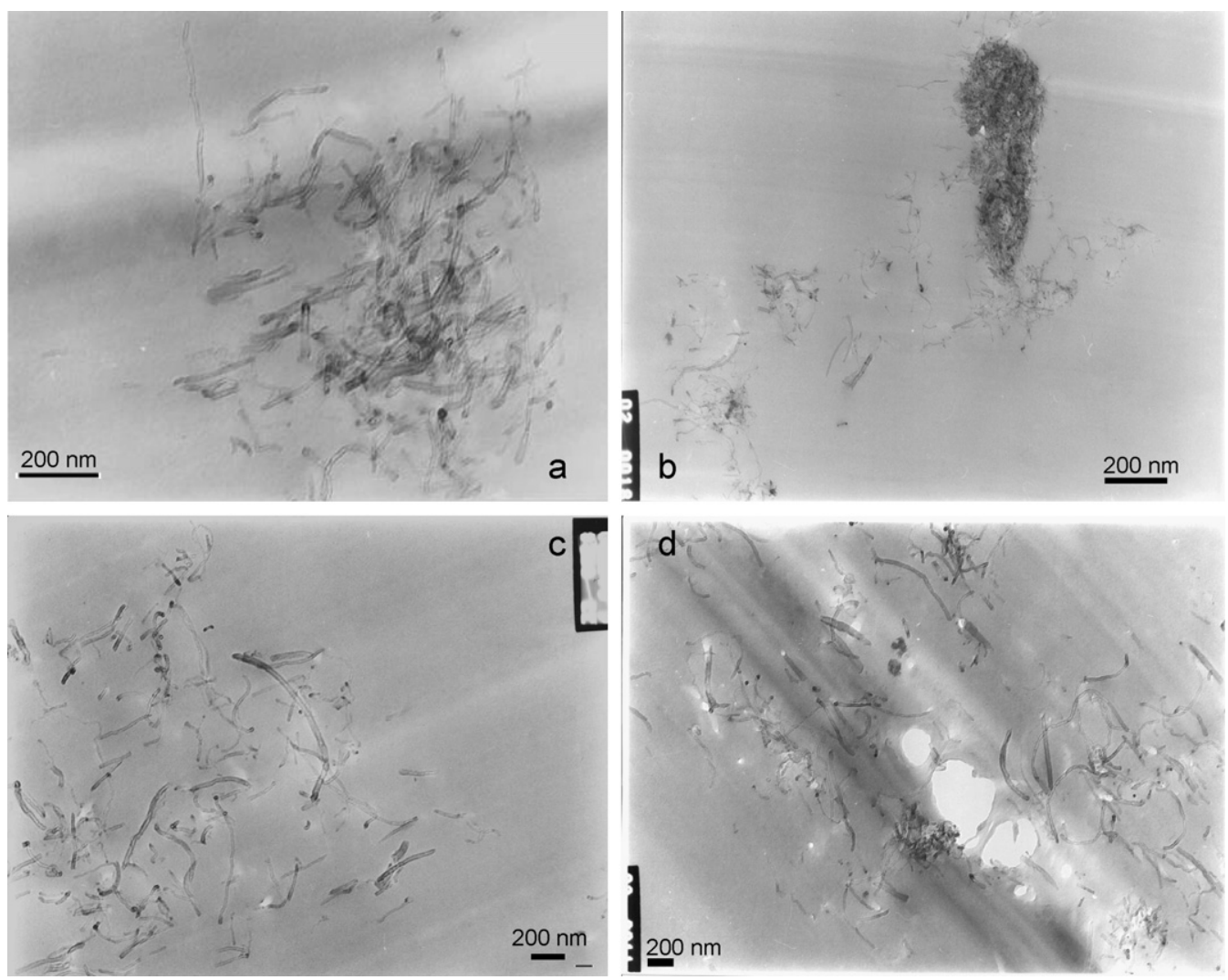

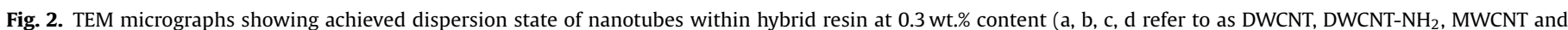
MWCNT- $\mathrm{NH}_{2}$, respectively.).

polyester resin. The gap size between the ceramic rolls was kept constant at $5 \mu \mathrm{m}$. The speeds of feed, center and apron rolls were set to 20,60 and $180 \mathrm{rpm}$, respectively. The dwell time of polyester/CNT suspension was about $2 \mathrm{~min}$. The collected CNT/polyester resin suspensions were then blended with vinyl-ester resin at a weight ratio of $1 / 3$, using a high speed mechanical stirrer for about $30 \mathrm{~min}$. Following addition of the styrene emission agent ( $1 \mathrm{wt} . \%$ ) to the prepared mixture, CoNAP and MEKP were introduced into the resultant system at a ratio of 0.2 and $1 \mathrm{wt} . \%$, respectively, to polymerize the hybrid resin system. Subsequently, the catalyzed resin suspensions were allowed to cure at room temperature followed by post-curing at $120^{\circ} \mathrm{C}$ for $2 \mathrm{~h}$. Please note that a special resin blend was used to manufacture nanocomposites. This is because, when employing commercial styrene containing polyester or vinyl-ester resins, heat evolved between the rotating rolls leads to instant styrene evaporation, thus increasing the viscosity of the resin left over the rolls. Therefore, to avoid styrene evaporation, the CNTs were first dispersed in a specially synthesized styrene-free polyester resin. Note that the viscosity of the corresponding polyester resin was intentionally considered high enough to apply as high shear rates as possible within gaps of the rolls to effectively break up the agglomerates and bundles of nanotubes within the intentional dwell time.

\subsection{Mechanical characterization of nanocomposites}

Tensile mechanical properties of the nanocomposites were determined according to DIN EN ISO 527.1. Dog bone shape specimens were prepared by countersinking through Mutronic dear drive 2000. The tensile samples were tested on Zwick Universal testing machine 1445 at a crosshead speed of $1 \mathrm{~mm} / \mathrm{min}$. The elongation of the specimen during the test was measured by means of distance encoder with a gauge length of $5 \mathrm{~mm}$.
The fracture toughness of the nanocomposites was measured according to ASTM-D 5045-96. Compact tension (CT) specimens were prepared in the same dear drive machine and tested at a crosshead speed of $1.3 \mathrm{~mm} / \mathrm{min}$., employing the universal testing machine with a $500 \mathrm{~N}$ load cell. The deformation of the specimens was monitored by detecting the crack opening displacement with the encoder. A sharp incipient crack was accomplished watchfully by hammering a razor blade into the corresponding notch. Six specimens of each sample were tested for statistical evaluation. The fracture energy $G_{k}$ was calculated based on the equation below.

$G_{I c}=\frac{K_{I c}^{2}}{E}$

In the above equation, $K_{I c}$ is the fracture toughness and calculated as an average of values for each batch and $E$ is the elastic modulus of each corresponding nanocomposite.

\subsection{Microscopic investigations}

TEM was utilized to qualitatively determine the dispersion state of the CNTs with and without $\mathrm{NH}_{2}$ functional groups within the hybrid polymer at nano-scale. TEM studies were performed by using a Philips EM 400 at $120 \mathrm{kV}$ acceleration voltages on the films of each sample in $30-100 \mathrm{~nm}$ thickness prepared by ultra-microtome cutting at room temperature. Philips SEM at $3 \mathrm{kV}$ voltages was also used to examine CNT induced macro-scale fracture failure modes that occurred within specimens under mechanical loading. Accordingly, the effects of CNTs with and without $\mathrm{NH}_{2}$ functional groups on the damage mechanisms of the nanocomposites were evaluated. 


\section{Results and discussion}

\subsection{Dispersion of nanotubes within polymer matrix}

Fig. 2a-d is the TEM micrographs showing achieved dispersion of DWCNT, DWCNT-NH $\mathrm{N}_{2}$, MWCNT and MWCNT-NH $\mathrm{H}_{2}$ at $0.3 \mathrm{wt} . \%$ loading rate within the corresponding hybrid polymer resin, respectively. MWCNT and MWCNT- $\mathrm{NH}_{2}$ exhibited relatively good dispersion within the hybrid polymer without possessing any significant dense agglomerates. However, in the case of DWCNT and DWCNT- $\mathrm{NH}_{2}$, some agglomerated zones were visible within the hybrid resin. DWCNTs tend to form relatively high fraction of agglomerates within the resin system due to their relatively high surface area. $\mathrm{NH}_{2}$ functional groups over the surfaces of CNTs are expected to improve not only the final dispersion state of CNTs within the resin matrix, but also the chemical interactions at the interface. This subject of interest will be handled in the following titles.

\subsection{Evaluation of tensile mechanical properties}

Fig. 3 gives the ultimate tensile strength (UTS) of the nanocomposites containing various types of CNTs (MWCNT, MWCNT-NH $\mathrm{N}_{2}$, DWCNT and DWCNT- $\mathrm{NH}_{2}$ ) with respect to filler content. It was found that there is no significant effect of CNT addition on the UTS of the polymer system studied. In the literature, a number of studies [1-6] have pointed out that use of CNTs with high specific surface area (SSA) and aspect ratio lead to improved tensile strength and fracture toughness for epoxy resin based nanocomposites. However, the same profound effect of CNTs is not likely to occur in resins polymerized via free radicals such as vinyl-ester and polyester. This is due to the fact that free radicals generated by decomposition of initiator (MEKP) added to the resin system could be easily entrapped within the galleries of CNTs. In this case, use of lower amount of MEKP than the required for the given amount of resin would result in heterogeneous polymerization across the part, with the final part having a variant cross-linking density distribution.

Furthermore, post-curing subsequently applied at elevated temperature causes the built-in thermal residual stresses to take place

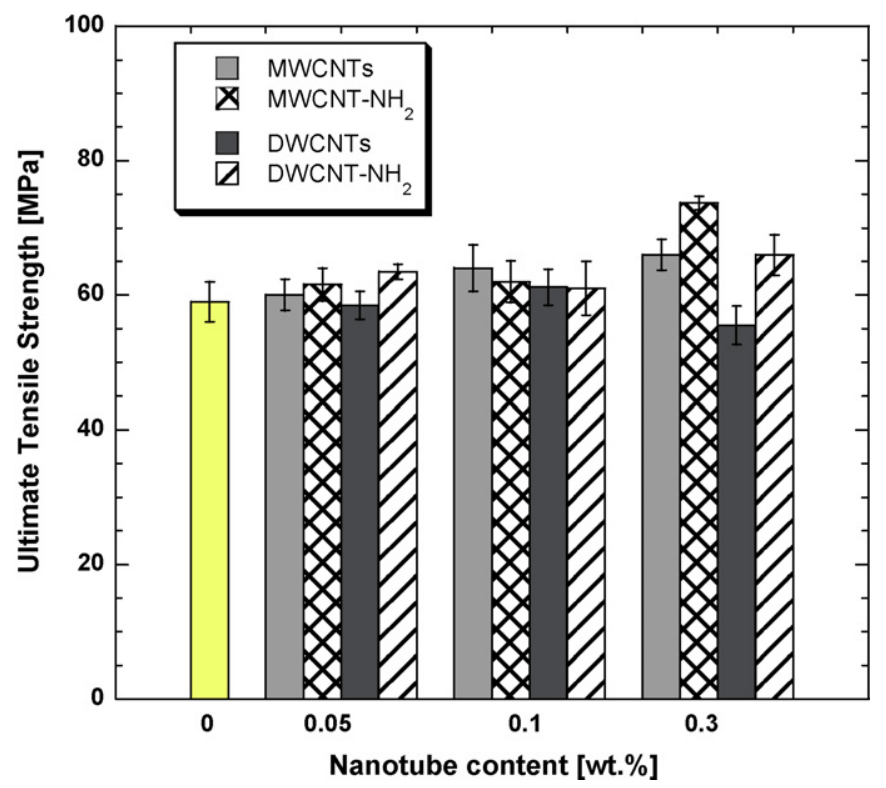

Fig. 3. Ultimate tensile strength of the nanocomposites containing MWCNT, MWCNT- $\mathrm{NH}_{2}$, DWCNT and DWCNT- $\mathrm{NH}_{2}$ with respect to nanotube content. within the composite parts, which induces voids and micro-cracks preferably located nearby or around the zone of agglomerated CNTs within the nanocomposites. This may reduce especially the mechanical strength of the resulting nanocomposites. Similarly, Peng et al. [17] reported that single walled CNTs are able to effectively trap free radicals during room temperature polymerization, thus leading to relatively low cross-linking density across the part. In our case, this is somewhat more pronounced for nanocomposites containing DWCNTs. This is because DWCNTs exhibit relatively high surface area that leads to relatively high tendency to form relatively large agglomerates. Therefore, one could presume that the more amounts of free radicals may have probably entrapped within the galleries of DWCNTs than within the galleries of MWCNTs. This is because DWCNTs occupy higher volume within the resin system than MWCNTs at a given weight percentage. On the other hand, the amino functional groups over the surfaces of CNTs are expected to improve their dispersion state within the resin matrix by increasing their surface polarity and by enhancing the chemical interactions at the interface between CNTs and the surrounding matrix resin. This accounts for the fact that nanocomposites containing amino functionalized nanotubes exhibit higher tensile strength as compared to nanocomposites with untreated ones.

Fig. 4a and b gives the elastic modulus values of the nanocomposites with various types of CNTs (MWCNT, MWCNT-NH $\mathrm{N}_{2}$, DWCNT and DWCNT- $\mathrm{NH}_{2}$ ). The average modulus value for the neat polymer was measured to be about $2400 \mathrm{MPa}$. In brief, incorporation of MWCNT-NH $\mathrm{H}_{2}$ and MWCNTs into the hybrid resin was found to lead to higher elastic modulus values in the resulting nanocomposites. Elastic moduli of the nanocomposites with MWCNT-NH and MWCNTs at $0.1 \mathrm{wt} . \%$ content are about 18 and 15\% higher than of neat hybrid polymer, respectively. However, addition of similar contents of DWCNTs or DWCNT- $\mathrm{NH}_{2}$ has no substantial effect on the modulus values. In a similar manner, Gryschuck et al. [12] investigated the mechanical and thermal properties of vinyl-ester based nanocomposites prepared with MWCNTs. They reported that the aspect ratio of the CNTs may have substantial effects on the chemical interactions at the interface, and that cure kinetics of the resin may vary with regard to volume content of CNTs that the matrix resin contains. Note that the $\mathrm{NH}_{2}$ functionalized CNTs used in our study were functionalized using ball milling, during which CNTs lose their strength partially via their broken carbon-carbon bonds and their aspect ratio is reduced. At this stage, one can expect drastically lower elastic modulus values from nanocomposites containing MWCNT- $\mathrm{NH}_{2}$ than those with MWCNTs. However, nanocomposites with MWCNT- $\mathrm{NH}_{2}$ at each given loading rate possessed significantly relatively high elastic modulus values, just as in the same manner as tensile strength. This is ample evidence that $\mathrm{NH}_{2}$ functional groups enhance the adhesion at the interface between the CNTs and the polymer matrix while assisting in improving dispersion of CNTs within the resin system without interfering with double carbon bonds of polymer chains that are supposed to react with styrene. Our previous study [11] revealed that resin suspensions containing MWCNTs and MWCNT$\mathrm{NH}_{2}$ showed similar rheological response, despite five times lower aspect ratio of MWCNT- $\mathrm{NH}_{2}$ than of MWCNTs. This implies the effectiveness of $\mathrm{NH}_{2}$ functional groups in homogenous dispersion of CNTs. Moreover, the Halphin-Tsai equation for random orientation of short fibers within composites was utilized in order to have better insight to the reinforcement mechanism of CNTs with and without $\mathrm{NH}_{2}$ functional groups within the corresponding hybrid resin. Note that these corresponding equations presume that short fibers with circular cross-section posses a perfect distribution in and very well impregnation with the polymer matrix resin in which they are embedded. Based on these assumptions, longitudinal and transverse moduli of unidirectional discontinuous fiber reinforced 

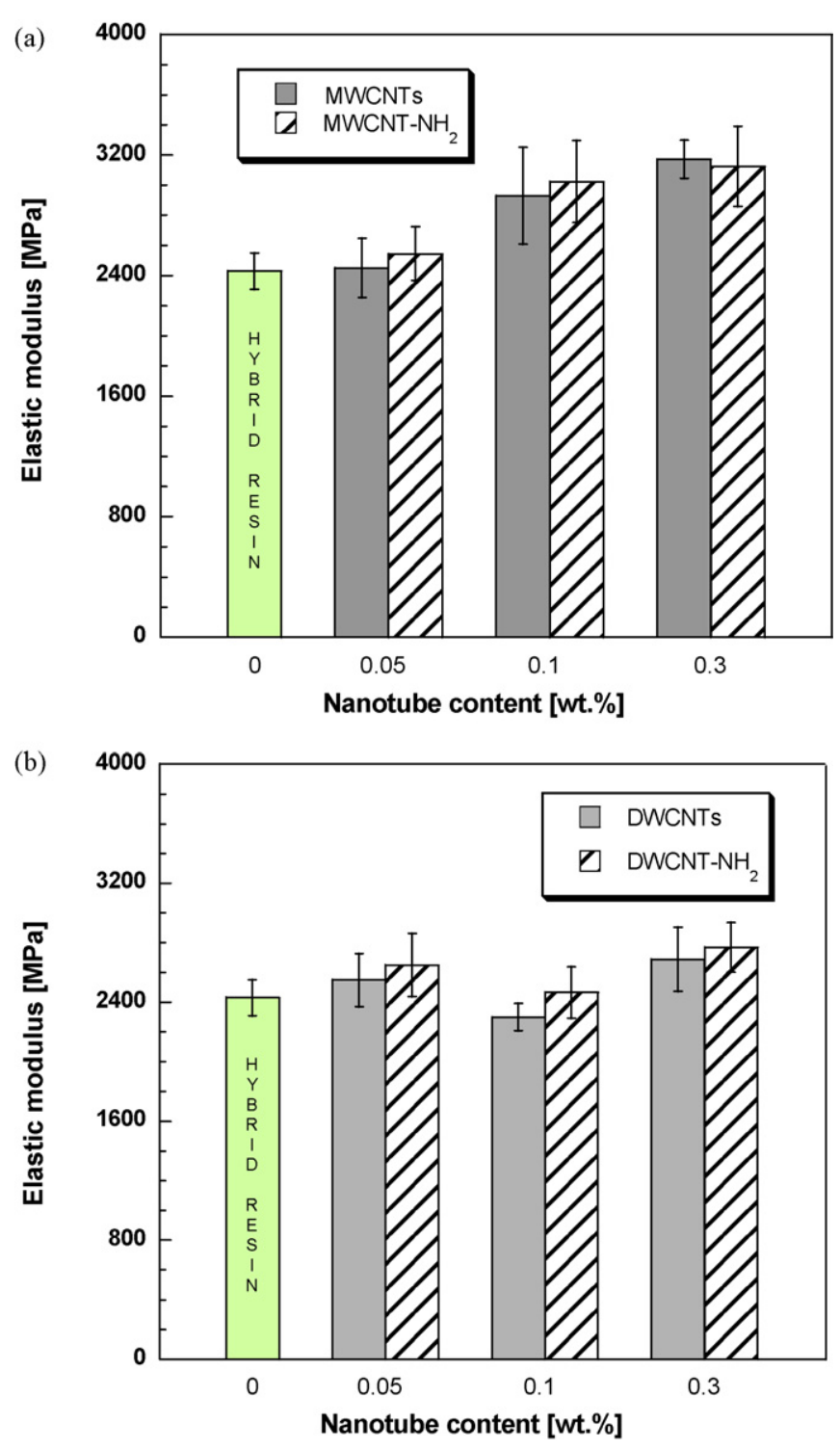

Fig. 4. Elastic modulus of the nanocomposites with respect to nanotube content (a) with MWCNT and MWCNT-NH $\mathrm{H}_{2}$ and (b) with DWCNT and DWCNT-NH $\mathrm{N}_{2}$.

composites are computed, using Eqs. (2) and (3), respectively [18].

$E_{11}=\frac{1+2\left(l_{f} / d_{f}\right) \eta_{L} v_{f}}{1-\eta_{L} v_{f}} E_{m}$

$E_{22}=\frac{1+2 \eta_{T} v_{f}}{1-\eta_{T} v_{f}} E_{m}$

Where

$\eta_{L}=\frac{\left(E_{f} / E_{m}\right)-1}{\left(E_{f} / E_{m}\right)+2\left(l_{f} / d_{f}\right)}$

$\eta_{T}=\frac{\left(E_{f} / E_{m}\right)-1}{\left(E_{f} / E_{m}\right)+2}$

Composite with randomly oriented discontinuous fibers shows planar isotropic behavior. For such a composite, the tensile modulus can be calculated using the below equation.

$E_{\text {composite }}=\frac{3}{8} E_{11}+\frac{5}{8} E_{22}$

All these above equations were then adopted to predict elastic modulus of nanocomposites with different types of carbon nan-

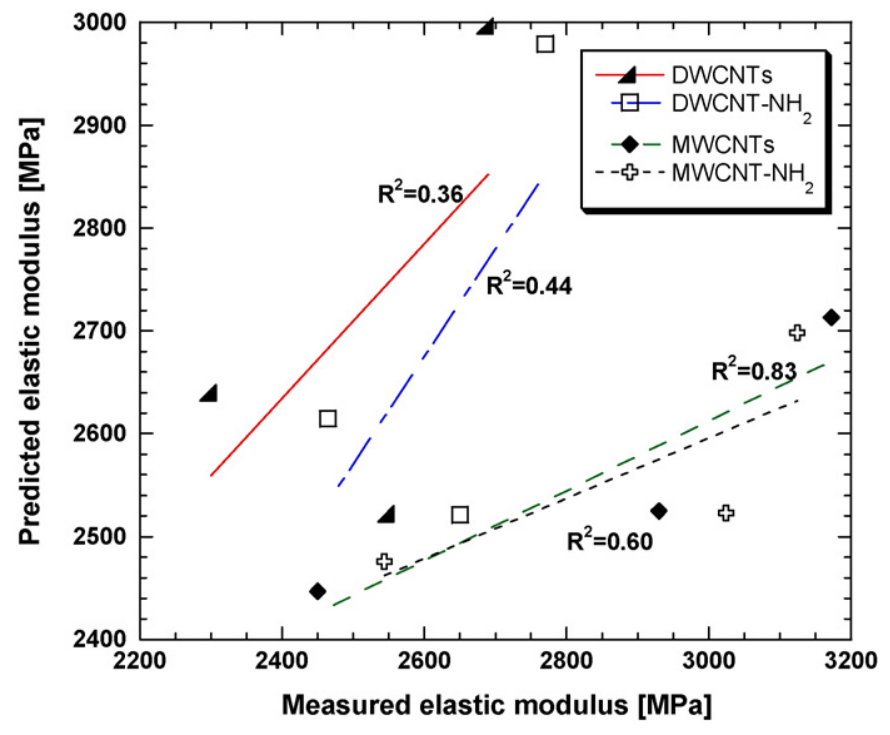

Fig. 5. Predicted elastic modulus values with respect to measured elastic modulus at different contents of CNTs.

otubes. Here, $E_{\text {composite }}$ is the elastic modulus of the nanocomposite, $l_{f}$ is the length of corresponding carbon nanotubes, $d_{f}$ is the average outer diameter of carbon nanotubes, $E_{f}$ is the elastic modulus of the nanotubes ( 1 TPa for each type of CNTs), $E_{m}$ is the modulus of the hybrid resin, and $v_{f}$ is the volume content of nanotubes. Please note that the volume content of the nanotubes is indeed difficult to calculate because of its dependence on the density, which is also dependent on the diameter distribution and defects of nanotubes. In this manner, we employed an approach suggested by Thostenson and Chou [15] to calculate the density of CNTs $\left(\rho_{\text {CNT }}\right)$.

$\rho_{C N T}=\frac{\rho_{g}\left(d_{a}^{2}-d_{i}^{2}\right)}{d_{a}^{2}}$

The equation above considers the inner and outer diameters of CNTs $\left(d_{i}\right.$ and $\left.d_{a}\right)$ and the density of graphite $\left(\rho_{g}\right)$. The density of hybrid resin was calculated based on the rule of mixture and taken as $1.13 \mathrm{~g} / \mathrm{cm}^{3}$ in the calculations. Density of the graphite was considered to be $2.25 \mathrm{~g} / \mathrm{cm}^{3}$. Accordingly, volume fraction of each type of carbon nanotubes was computed.

Fig. 5 gives the experimentally obtained and predicted values of elastic modulus of the nanocomposites containing DWCNTs and MWCNTs with and without functional groups at the specified weight contents $(0.05,0.1$ and $0.3 \mathrm{wt} . \%)$. Based upon the statistical $R^{2}$ values of the linear regression, nanocomposites with MWCNTs $(0.83)$ and MWCNT-NH$H_{2}(0.60)$ have better agreement between the predicted and experimentally measured elastic moduli values than do nanocomposites with DWCNTs (0.36) and DWCNT-NH (0.44). Hence, proportional to experimental findings, MWCNTs and MWCNT- $\mathrm{NH}_{2}$ were theoretically proved to have better dispersion within hybrid polymer compared to DWCNTs and DWCNT- $\mathrm{NH}_{2}$ as well. In brief, the results obtained showed that MWCNTs and MWCNT- $\mathrm{NH}_{2}$ reinforced vinyl-ester-polyester based nanocomposites featured better tensile mechanical properties as compared to those with DWCNTs and DWCNT-NH 2 . Also, aspect ratios, SSA, volume content, and $\mathrm{NH}_{2}$ functional groups over the surfaces of CNTs are particularly crucial to the final degree of polymerization to some extent.

\subsection{Fracture toughness of nanocomposites}

Figs. 6 and 7 give the fracture toughness values of nanocomposites prepared with various types and amounts of CNTs 


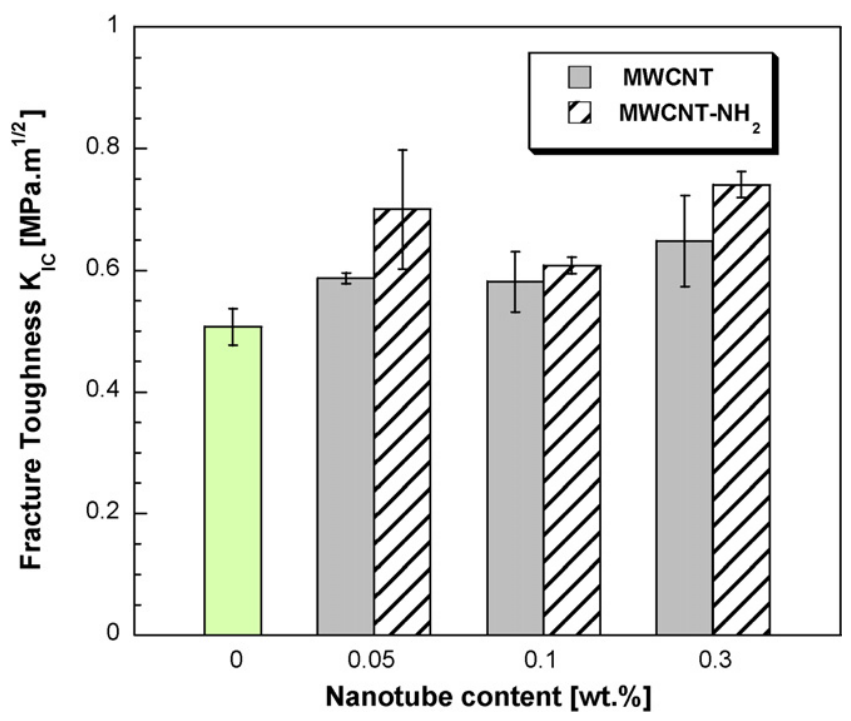

Fig. 6. Fracture toughness values of nanocomposites with MWCNT and MWCNT$\mathrm{NH}_{2}$ with respect to nanotube content.

including MWCNTs, MWCNT-NH 2 , DWCNTs and DWCNT-NH $\mathrm{N}_{2}$. The matrix polymer exhibited a fracture toughness value of about $0.5 \mathrm{MPa} \mathrm{m}^{1 / 2}$. At each concentration, nanocomposites with MWCNTs and MWCNT- $\mathrm{NH}_{2}$ exhibited higher fracture toughness values than those with DWCNTs and DWCNT- $\mathrm{NH}_{2}$. Moreover, nanocomposites with MWCNT- $\mathrm{NH}_{2}$ exhibited high fracture toughness values relative to those with MWCNTs. Incorporation of $0.3 \mathrm{wt} . \%$ of MWCNT- $\mathrm{NH}_{2}$ resulted with the highest improvement (by 40\%) on the toughness value compared to neat hybrid polymer. Addi-

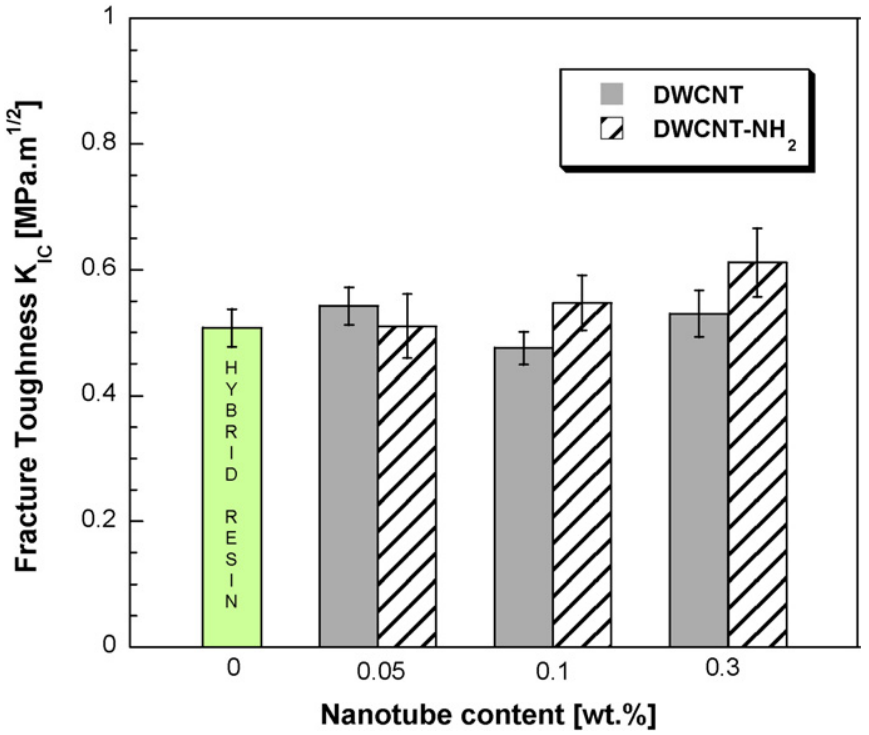

Fig. 7. Fracture toughness values of nanocomposites with DWCNT and DWCNT-NH with respect to nanotube content.

tion of DWCNTs with and without $\mathrm{NH}_{2}$ functional groups has almost no significant influence on the fracture toughness value of their resulting nanocomposites. Table 2 gives the calculated fracture energy of nanocomposites with various types of CNTs. Note that fracture energy of the neat hybrid polymer is $105.7 \mathrm{~J} / \mathrm{m}^{2}$. As seen in the table, the highest (almost twice of neat hybrid polymer) and the lowest energies were obtained from nanocomposites with MWCNT- $\mathrm{NH}_{2}$ and DWCNTs, respectively. These results are
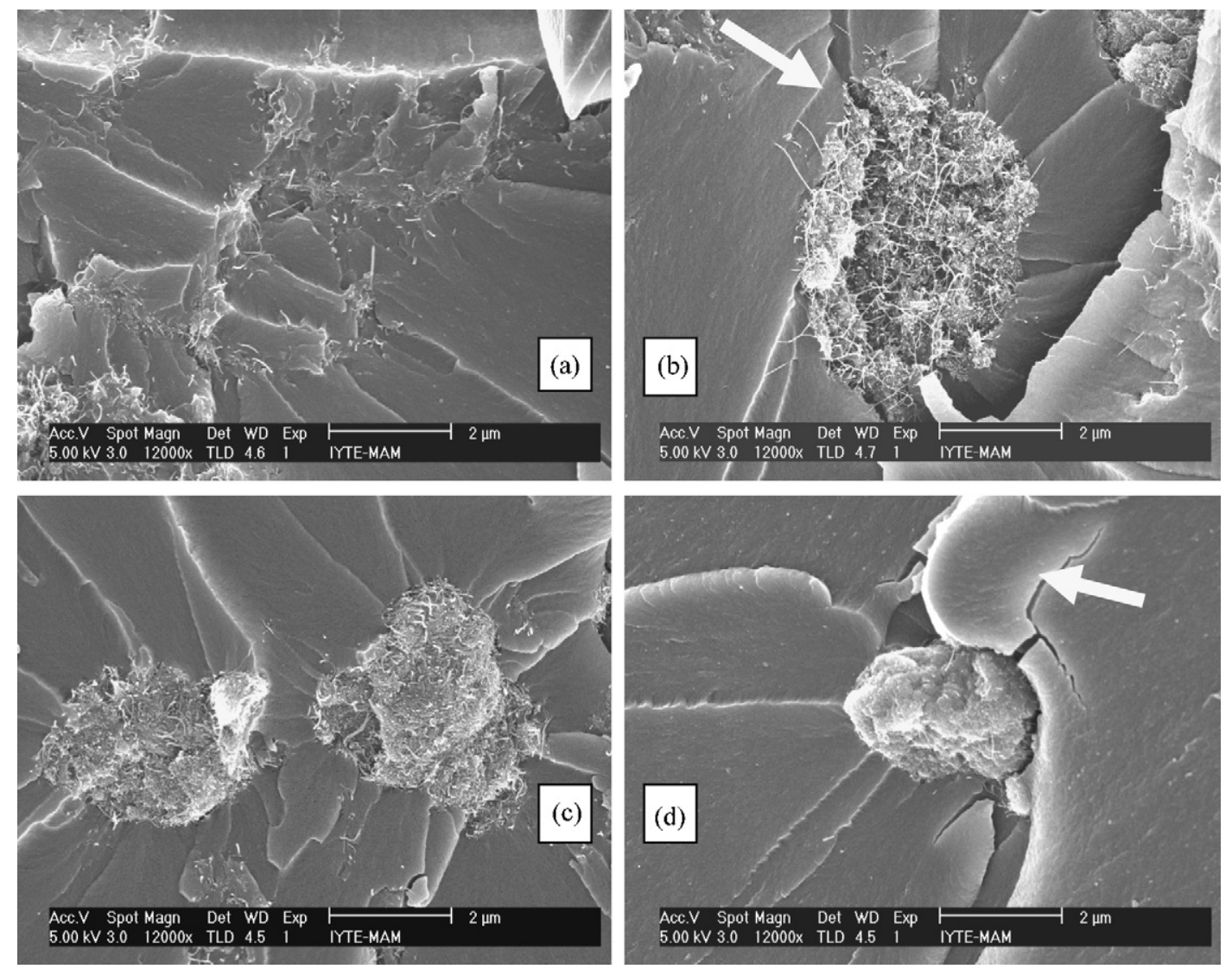

Fig. 8. SEM photos of tensile fracture surface of nanocomposites with (a) MWCNT, (b) MWCNT- $\mathrm{NH}_{2}$, (c) DWCNT and (d) DWCNT-NH 2 at 0.3 wt.\% CNT content. 
Table 2

Calculated fracture energy of each corresponding nanocomposite $\left(\mathrm{J} / \mathrm{m}^{2}\right)$.

\begin{tabular}{lllcc}
\hline Weight\% & MWCNTs & MWCNT-NH & DWCNTs & DWCNT-NH \\
\hline 0.05 & 140.5 & 197.6 & 114.3 & 98.15 \\
0.1 & 114.8 & 119.1 & 96.3 & 121.3 \\
0.3 & 129.1 & 172.5 & 104.1 & 134.3 \\
\hline
\end{tabular}

Neat hybrid resin $=105.7$.

consistent with those achieved in tensile mechanical response of their matching nanocomposites. Nanotubes with their huge aspect ratio are supposed to show fiber-like behavior. As discussed earlier, partially agglomerated morphology was in general observed for each type of CNTs, especially for DWCNTs due to their relatively high SSA. Gojny et al. [2-4] concluded that large surface areas lead to a more efficient improvement of fracture toughness, and that improper impregnated agglomerates act as defects in the epoxy based nanocomposites. On the behalf of the experimental results obtained, they further suggested that a combination of well dispersed nanotubes with well impregnated smaller agglomerates is the most promising state of nanofiller dispersion, thus resulting in a substantial enhancement of the fracture toughness. However, this corresponding approach may not be entirely valid for our case. Unlikely, as elucidated under previous sections, nanofillers with high surface area seems to be not appropriate for resins to be polymerized via free radicals. In brief, the larger SSA of CNTs, the more probable it is for radicals to be trapped within galleries of nanotubes, which lowers the degree of polymerization while reducing the cross-linking density. Our approach shows great consistency with the experimental findings obtained in this study. The fracture surfaces of the failed tensile and fracture test specimens are in this respect worth examining to relate the CNT induced fracture mechanisms and failure modes to the fracture behavior of the resultant nanocomposites. Fig. 8a-d is the SEM tensile fracture surfaces of the nanocomposites prepared with $0.3 \mathrm{wt} . \%$ of MWCNTs, MWCNT$\mathrm{NH}_{2}$, DWCNTs and DWCNT- $\mathrm{NH}_{2}$, respectively. As seen in the photo (Fig. 8a), MWCNTs have homogenous dispersion with very few separate agglomerates in the surrounding matrix resin. However, DWCNTs (Fig. 8c) exhibited relatively dense large agglomerates and few individuals around them. In fact, this trend is expected because DWCNTs have relatively high surface area. Therefore, hypothetically more energy is needed for DWCNTs than for MWCNTs to disperse their agglomerates into individual ones via 3-roll milling. On the other hand, the matrix cracking passing through the mid-plane of agglomerates of MWCNT- $\mathrm{NH}_{2}$, as indicated by a white arrow on the photo (Fig. 8b), is observable. Moreover, agglomerates of DWCNT$\mathrm{NH}_{2}$ (Fig. 8d) exhibited, on the other hand, relatively weak adhesion to the matrix resin in such a way that extensive de-bonding and incurable matrix cracking that occur around the corresponding agglomerates (as indicated by white arrow) are highly noticeable. The difference apparent in the fracture modes of nanocomposites containing MWCNT and DWCNT with and without amine functional groups can be reasonably considered remarkable evidence for the CNT induced altered resin chemistry around local CNT-rich matrix resin, depending on the type, in other words, on the different surface area and aspect ratio of CNTs. Please note that SEM photos showing the dense agglomerates of amino functionalized nanotubes were intentionally chosen to associate the mechanical properties of the nanocomposites with their curing behavior. In fact, these observations showed that amine functional groups, degree of nanotube agglomeration, size of the nanotube agglomerates and their distribution within the surrounding matrix are vastly critical to the ultimate response of the nanocomposites.

However, curing behavior of resin suspensions with carbon nanotubes is beyond the scope of this paper and will be addressed elsewhere soon. Fig. 9a and $\mathrm{c}$ is the SEM fracture surfaces of the
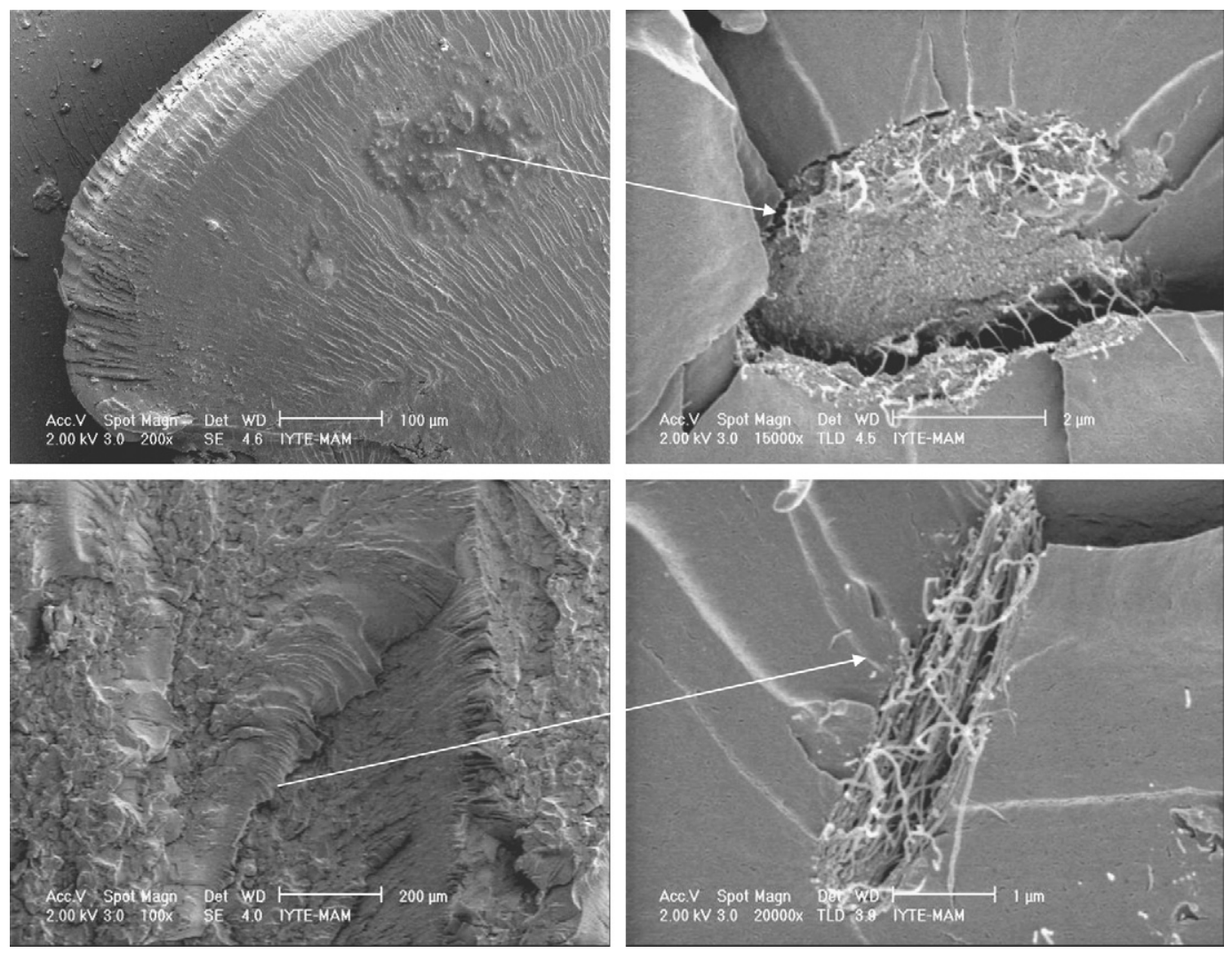

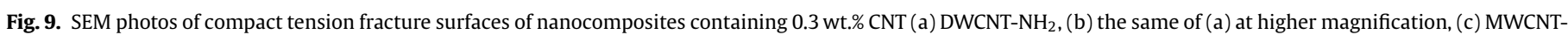
$\mathrm{NH}_{2}$, and (d) the same of (c) at higher magnification. 
compact tension (CT) composite specimens prepared with $0.3 \mathrm{wt} . \%$ of DWCNT- $\mathrm{NH}_{2}$ and MWCNT-NH $\mathrm{N}_{2}$, respectively. Fig. 9b and d shows the magnified zone indicated by the arrows highlighted in Fig. 9a and c, respectively. The same trend as in tensile fracture behavior of nanocomposites is observable. In terms of fracture mechanics, dense agglomerates act probably as defects, thus triggering void nucleation and/or micro-crack coalescences at the interface between CNTs and the surrounding matrix resin. Further, the characteristics of the bulk matrix may also play a crucial role in overall response of the entire nanocomposite part. In other words, one could hypothetically conclude that the property of the bulk matrix around the larger agglomerates may be locally different from the overall response of the entire nanocomposite dependent on the type of CNTs and amine functional groups. This is because the amount of free radicals trapped within galleries of CNTs is highly dependent on the physical properties of CNTs, including aspect ratio and surface area. This would alter the array of crack paths across the corresponding part prior to its failure under load. On the other hand, based on SEM examination, it is reasonable to make an overall statement that, besides altered resin chemistry associated with the extent of free radicals available to initiate polymerization reaction depending on different characteristics of CNTs, nanotube agglomeration itself, especially in the case of DWCNTs, might have been, moreover, another major cause of lower than the expected mechanical properties obtained from the nanocomposites. In brief, all these issues mentioned above could explain the reason of the anomalous trend observed for the tensile mechanical properties and fracture toughness of the nanocomposites as a function of weight content of CNTs with and without amine functional groups.

\section{Conclusions}

In this study, tensile mechanical behavior and fracture toughness of vinyl-ester-polyester hybrid resin system containing low content of MWCNTs, MWCNT-NH $\mathrm{N}_{2}$, DWCNTs and DWCNT-NH $\mathrm{N}_{2}$ was investigated. The CNTs were dispersed within the matrix system via 3-roll milling. It was found that, regardless of type or content of CNTs, tensile strength of the resulting nanocomposites was found not to be significantly affected by the addition of CNTs. It was also revealed that nanocomposites containing MWCNTs and MWCNT$\mathrm{NH}_{2}$ resulted in higher tensile modulus, fracture toughness and fracture energy values compared to nanocomposites prepared with DWCNTs or DWCNT- $\mathrm{NH}_{2}$. Moreover, when incorporated into free radically polymerized thermosetting resins such as polyester or vinyl-ester, reinforcing efficiency of DWCNTs and DWCNT-NH 2 was shown to be lower compared to that of MWCNTs and MWCNT-NH 2 . The rationale behind this major statement is based on the number of free radicals accessible to the polymerization reaction. As the surface area of CNTs increase, more free radicals are expected to be entrapped within the galleries of CNTs. In brief, the availability of free radicals within the polymer system used as well as extent and tendency of CNT agglomeration are highly associated with the observed mechanical properties of the resulting nanocomposites. In the future work, curing behavior of the CNT modified resin suspensions will be intensively studied and discussed in order to gain better insight into the effects of radical entrapment and degree of CNT agglomeration on the mechanical properties of the resulting nanocomposites reported in this study.

\section{Acknowledgements}

The authors acknowledge TUBITAK of Turkey and JULICH of Germany for financial support.

\section{References}

[1] F.H. Gojny, K. Schulte, Compos. Sci. Technol. 34 (2004) 2303-2308

[2] F.H. Gojny, M.H.G. Wichmann, B. Fiedler, K. Schulte, Compos. Sci. Technol. 65 (2005) 2300-2313.

[3] F.H. Gojny, J. Nastalczyk, Z. Roslaniec, K. Schulte, Chem. Phys. Lett. 370 (2003) 820-825.

[4] F.H. Gojny, M.H.G. Wichmann, U. Köpke, B. Fiedler, K. Schulte, Compos. Sci. Technol. 34 (2004) 2363-2371.

[5] B. Fiedler, F.H. Gojny, M.H.G. Wichmann, M. Nolte, K. Schulte, Compos. Sci. Technol. 66 (2006) 3115-3125.

[6] Frankland, A. Caglar, D.W. Brenner, M. Griebe, J. Phys. Chem. B 106 (2002) 3046-3048.

[7] J.A. Kim, D.G. Seong, T.J. Kang, J.R. Youn, Carbon 44 (2006) 1898-1905.

[8] Z. Yaping, Z. Aibio, C. Quingha, Z. Jiaoxia, N. Rongchnag, Mater. Sci. Eng. A 435 (2006) 145-149.

[9] M. Grujicic, Y.P. Sun, K.L. Koudela, Appl. Surf. Sci. 253 (2007) 3009-3021.

[10] A.T. Seyhan, F.H. Gojny, M. Tanoglu, K. Schulte, Eur. Polym. J. 43 (2007) 374-379.

[11] A.T. Seyhan, F.H. Gojny, M. Tanoglu, K. Schulte, Eur. Polym. J. 43 (2007) 2836-2847.

[12] O. Gryschuck, J.K. Kocsis, R. Thomann, Z. Kanya, I. Kiricsi, Compos. Part A 37 (2006) 1252-1259.

[13] E.T. Thostenson, Z. Ren, T.W. Chou, Compos. Sci. Technol. 61 (2001) 1899-1912.

[14] E.T. Thostenson, L. Chunyiu, T.W. Chou, Compos. Sci. Technol. 65 (2005) 491516.

[15] E.T. Thostenson, T.W. Chou, J. Phys. D: Appl. Phys. 36 (2003) 573-582.

[16] E.T. Thostenson, T.W. Chou, Carbon 44 (2006) 3022-3029.

[17] H. Peng, P. Reverdy, V.N. Khabasheku, J.L. Margrave, J. Am. Chem. Soc. 125 (2003) 15174-15182.

[18] J.C. Halpin Affdl, J.L. Kardos, Polym. Eng. Sci. 16 (1976) 344-352. 